\title{
Limited seizures, but a broader impact
}

\section{Crises limitadas, mas impacto amplo}

Ana Carolina COAN ${ }^{1,2}$

1 Universidade Estadual de Campinas, Departamento de Neurologia, Serviço de Neurologia Infantil, Campinas SP, Brazil.

¿Universidade Estadual de Campinas, Instituto Brasileiro de Neurociência e Neurotecnologia, Campinas SP, Brazil.

ACC (iD https://orcid.org/00000002-2888-929X

Correspondence: Ana Carolina Coan; Email:acoan@unicamp.br.

Conflict of interest: There is no conflict of interest to declare.

Received on October 05, 2021; Accepted on October 07, 2021.

\section{(cc) BY}

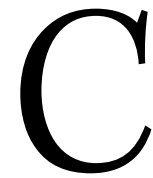

hildren with self-limited epilepsy with centrotemporal spikes (SeLECTS) usually have a low number of seizures during the evolution of the disorder, which tends to remit only a few years after the onset. Frequently, antiseizure medication might not even be considered due to the low seizure burden. However, even in this apparently mild disorder, epilepsy is much more than only seizures. Despite the easy-to-control seizures and the early remission, there is accumulated evidence that SeLECTS has a broader impact on children ${ }^{1}$. Neuropsychological profile of children with SeLECTS is lower when compared with age-matched controls, with reduced scores on memory, attention, cognitive flexibility, and verbal fluency ${ }^{1,2}$. Moreover, psychiatric comorbidities and reduced social cognition can also impact children with SeLECTS, at least during the active stage of the disorder ${ }^{3}$.

In this issue of Arquivos de Neuropsiquiatria, Bernardo et al. ${ }^{4}$ described another possible impact of SeLECTS. In this study, the authors investigated oromotor praxis in a large cohort of children with SeLECTS. They clearly show that these children have poorer performance in simple and sequenced oral gestures than age-matched controls. Although there are specific cases of Rolandic seizures associated with cognitive impairment and oral dyspraxia, the present study shows that even in a larger group of classical SeLECTS, oral praxis is not well developed as age-matched controls.

Neuroimaging studies of the function and organization of brain show abnormal gray matter density and brain connectivity in children with SeLECTS, especially in the bilateral perisylvian region, including the inferior frontal gyrus and the opercular area of the precentral gyrus. These regions are essential for motor programming and execution of the mouth and tongue ${ }^{5,6}$. These abnormal brain networks could justify the worse performance of oral praxis in this group.

Comorbidities in SeLECTS are often associated with the risk of interictal epileptiform discharges that are very frequent, especially in the early onset of epilepsy ${ }^{5}$. Although this is not the current practice, there is a debate on how eliminating interictal discharges from electroencephalogram could impact cognition and other comorbidities in children with SeLECTS. Similarly, it is not clear which mechanisms are related to the poor performance of oral praxis in this group. Although the impact of interictal epileptiform discharges may sound like an interesting hypothesis, we must remember that the use of antiseizure medication and possibly the presumed genetic etiology of the epilepsy might play a role in the oral motor praxis.

According to the brain development, oral praxis evolves with age. Could the distinct profile of oral motor praxis between children with SeLECTS and controls be related to a slower brain maturation in this group? And if that is the case, when and why this brain development is affected? The increased knowledge of comorbidities in SeLECTS is important for an appropriate management of these children. In the past couple of decades, more evidence of the existence of these comorbidities and how they impact the quality of life of children with epilepsy is available. However, many questions are still open. How does epilepsy impact brain development? How do these comorbidities evolve with time? How distinct aspects of epilepsy, such as the occurrence of interictal epileptiform discharges, use of antiseizure medication, or social deprivation, could contribute to these comorbidities?

Epilepsy has a significant impact in children, even when seizures are under control. Poorer oral motor praxis could also be a risk factor for children with SeLECTS. Therefore, a complete medical evaluation and appropriate management of SeLECTS-associated comorbidities are recommended. 


\section{References}

1. Hamiwka LD, Wirrell EC. Comorbidities in pediatric epilepsy: beyond “just" treating the seizures. J Child Neurol. 2009 Jun;24(6):734-42. https://doi.org/10.1177/0883073808329527

2. Deonna T, Zesiger P, Davidoff V, Maeder M, Mayor C, Roulet E. Benign partial epilepsy of childhood: a longitudinal neuropsychological and EEG study of cognitive function. Dev Med Child Neurol. 2000 Sep;42(9):595-603. https://doi.org/10.1017/ s0012162200001122

3. Hermann BP, Struck AF, Busch RM, Reyes A, Kaestner E, McDonald CR. Neurobehavioural comorbidities of epilepsy: towards a networkbased precision taxonomy. Nat Rev Neurol. 2021 Sep. https://doi. org/10.1038/s41582-021-00555-z
4. Bernardo HNSA, Miziara CSMG, Manreza MLG, Mansur LL. Oral dyspraxia in self-limited epilepsy with centrotemporal spikes: a comparative study with a control group. Arq Neuro-Psiquiatr. 2021;79(12):1076-83. https:// doi.org/10.1590/0004-282X-ANP-2021-0056

5. Xiao F, An D, Lei D, Li L, Chen S, Wu X, et al. Real-time effects of centrotemporal spikes on cognition in rolandic epilepsy: an EEG-fMRI study. Neurology. 2016 Feb;86(6):544-51. https://doi. org/10.1212/WNL.0000000000002358

6. Belton E, Salmond CH, Watkins KE, Vargha-Khadem F, Gadian DG. Bilateral brain abnormalities associated with dominantly inherited verbal and orofacial dyspraxia. Hum Brain Mapp. 2003 Mar;18(3):194-200. https://doi.org/10.1002/hbm.10093 